\title{
Article \\ Spin Dependent Transport through Driven Magnetic System with Aubry-Andre-Harper Modulation
}

\author{
Arpita Koley ${ }^{1}$, Santanu K. Maiti ${ }^{1, * \mathbb{C}}$, Judith Helena Ojeda Silva ${ }^{2,3}$ and David Laroze $^{4}$ (D) \\ 1 Physics and Applied Mathematics Unit, Indian Statistical Institute, 203 Barrackpore Trunk Road, \\ Kolkata 700108, India; arpitakoley94@gmail.com \\ 2 Grupo de Física de Materiales, Universidad Pedagógica y Tecnológica de Colombia, Tunja 150003, Colombia; \\ judith.ojeda@uptc.edu.co \\ 3 Laboratorio de Química Teórica y Computacional, Grupo de Investigación Química-Física Molecular y \\ Modelamiento Computacional (QUIMOL), Facultad de Ciencias, Universidad Pedagógica y Tecnológica de \\ Colombia, Tunja, Boyacá 150003, Colombia \\ 4 Instituto de Alta Investigación, CEDENNA, Universidad de Tarapacá, Casilla 7D, Arica 1000000, Chile; \\ dlarozen@uta.cl \\ * Correspondence: santanu.maiti@isical.ac.in
}

check for updates

Citation: Koley, A.; Maiti, S.K.; Ojeda Silva, J.H.; Laroze, D. Spin Dependent Transport through Driven Magnetic System with Aubry-Andre-Harper Modulation. Appl. Sci. 2021, 11, 2309 https://doi.org/10.3390/app11052309

Academic Editors: Roberto Zivieri and Linda Angela Zotti

Received: 29 December 2020

Accepted: 1 March 2021

Published: 5 March 2021

Publisher's Note: MDPI stays neutral with regard to jurisdictional claims in published maps and institutional affiliations.

Copyright: (c) 2021 by the authors. Licensee MDPI, Basel, Switzerland. This article is an open access article distributed under the terms and conditions of the Creative Commons Attribution (CC BY) license (https:// creativecommons.org/licenses/by/ $4.0 /)$.

\begin{abstract}
In this work, we put forward a prescription of achieving spin selective electron transfer by means of light irradiation through a tight-binding (TB) magnetic chain whose site energies are modulated in the form of well known Aubry-Andre-Harper (AAH) model. The interaction of itinerant electrons with local magnetic moments in the magnetic system provides a misalignment between up and down spin channels which leads to a finite spin polarization (SP) upon locating the Fermi energy in a suitable energy zone. Both the energy channels are significantly affected by the irradiation which is directly reflected in degree of spin polarization as well as in its phase. We include the irradiation effect through Floquet ansatz and compute spin polarization coefficient by evaluating transmission probabilities using Green's function prescription. Our analysis can be utilized to investigate spin dependent transport phenomena in any driven magnetic system with quasiperiodic modulations.
\end{abstract}

Keywords: spin polarization; magnetic chain with AAH modulation; light irradiation

\section{Introduction}

"Spintronics" has been an emerging field of research during the last two decades which involves manipulation of electron spin along with its charge [1-10]. Unlike conventional electronic devices, where spin degree of freedom is neglected, spin based ones are much superior in the context of functionalities, new applications, operations and power consumption. Nowadays, spintronics can be applied almost everywhere, from data storage to robotics, speed control and navigation, designing of single as well as parallel logic gates, computer and mobile games and precise detection of defective cells, to name only a few. For most of these functionalities, spin injection across an interface is one of the important prerequisites. However, the fact is that the injection efficiency is remarkably low in most of the cases [11,12]. Though the efficiency can be improved by some mechanisms, still it is far away from the desired limit. One possible route to circumvent this issue is the use of a "spin filter" [13-20].

The basic concept to have filtration effect or in a more simple way to say to get polarized spin current from a completely unpolarized one, relies on the misalignment of up and down spin channels. That can be made possible by considering any kind of spin dependent scattering factor. One of the most common scattering mechanisms is associated with spin-orbit (SO) coupling [21-31]. In usual solid state materials SO coupling gets two different functional forms, known as Rashba [32] and Dresselhaus [33] SO couplings. The first one is involved with asymmetry in confining potential and hence can be regulated 
externally, whereas the other one is associated with the bulk inversion asymmetry of the material and its strength cannot be monitored. Different SO coupled systems like molecules, semiconducting materials, tailor made systems etc., with two-, three- and even multi-terminal junction configurations have been taken into account to explore the characteristics of polarized currents [34-47]. For all these cases, especially for molecular systems the key limitation is that the SO coupling is too weak [48]. Because of this fact, large mismatch among the two spin channels is not possible which prohibits to have higher $\mathrm{SP}$ in a reasonable bias window.

On the other hand, due to the existence of large spin dependent scattering, magnetic materials exhibit high degree of SP compared to the SO coupled ones. Most commonly ferromagnetic (FM) materials [49,50] are used, though nowadays attention has also been paid in different kinds of antiferromagnetic (AFM) materials [51-56] for spin filtration. For efficient functioning, tuning of spin polarized current is extremely crucial and that is usually done by applying a magnetic field. However, it has several limitations especially for small size systems where confining a magnetic field is a challenging task. To eliminate this prescription some alternative proposals have been put forward by a few groups, including us. Placing the functional element within a suitable gate electrode, the degree of SP and its phase can be tuned selectively, and the gate controlled transport phenomena have also been discussed in some other contexts.

In the present work we follow a different scheme, probably not explored so far in literature, where SP is engineered by means of light irradiation [57-63]. To substantiate this fact we consider a one-dimensional (1D) FM system which is irradiated by an arbitrary polarized light (see Figure 1). Each site of the magnetic chain is associated with a local magnetic moment that interacts with the injected electron spin [64-69]. Because of this, interaction electrons get scattered. The up and down spin energy channels are largely modified due to irradiation as it renormalizes the hopping strength, and this fact is directly reflected into the transport behavior. To make the system more realistic we introduce disorder in the proposed model. Instead of "uncorrelated" disorder, we consider a "correlated" one in the form of Aubry-Andre-Harper model [70-77] since the later one exhibits several atypical signatures. Both diagonal, off-diagonal and generalized AAH systems have been extensively studied in literature exploiting several unusual phenomena, especially along the line of electronic localization, due to unique and diverse characteristic features of AAH models, and here in our present work we concentrate only on diagonal AAH system as a first attempt and discuss the interplay between the AAH potential and irradiation on spin selective electron transmission.

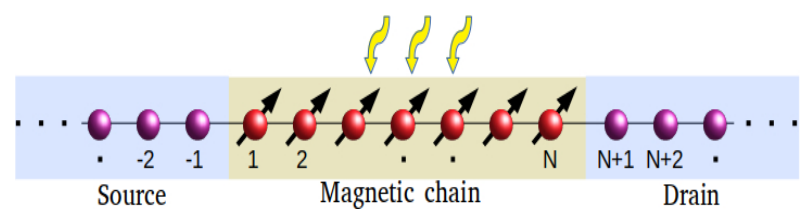

Figure 1. Spin polarized setup where a one-dimensional magnetic chain is coupled to non-magnetic source and drain electrodes. The magnetic chain is subjected to light irradiation which controls spin transfer through the junction.

Describing the quantum system within a tight-binding framework where irradiation effect is incorporated via the usual Floquet prescription [59,60], we determine spin dependent transmission probabilities following the Green's function formalism [78-82]. Using the transmission probabilities, we evaluate spin polarization coefficient. From the results, we find that the degree along with the phase of SP can be tuned in a wide range by means of irradiation. Several interesting features are emerged and our results might be useful in designing spin based electronic devices in near future.

The rest of the work is arranged as follows: In Section 2 we illustrate the spin polarized setup and TB Hamiltonian, and then give an outline of theoretical prescription for studying 
spin dependent transport phenomena. All the results are presented and critically analyzed in Section 3. Finally, we summarize our essential findings in Section 4.

\section{Magnetic Junction, TB Hamiltonian and Theoretical Formulation}

\subsection{Junction Setup and the Hamiltonian}

Let us begin with the spin polarized setup, shown in Figure 1, where a magnetic chain having $N$ lattice sites (filled red balls) is clamped between two non-magnetic (NM) electrodes, commonly referred as source (S) and drain (D). Each site of the magnetic chain contains a finite magnetic moment which interacts with the injected spin $\sigma(\sigma=\uparrow, \downarrow)$. The magnetic chain is subjected to light irradiation (yellow arrows) that plays the central role for engineering spin polarization in our analysis.

The Hamiltonian of the magnetic nanojunction can be written as

$$
H=H_{m a g}+H_{S}+H_{D}+H_{t u n}
$$

where different sub-Hamiltonians are associated with different parts of the junction. We describe explicitly all these terms one by one in TB framework as follows.

The sub-Hamiltonian $H_{m a g}$ reads as

$$
H_{\text {mag }}=\sum_{i} c_{i}^{\dagger}\left(\epsilon_{i}-\vec{h}_{i} \cdot \vec{\sigma}\right) c_{i}+\sum_{i}\left(c_{i+1}^{\dagger} \tilde{t} c_{i}+\text { h.c. }\right)
$$

where $c_{i}^{\dagger}=\left(\begin{array}{cc}c_{i \uparrow}^{\dagger} & c_{i \downarrow}^{\dagger}\end{array}\right) \cdot c_{i \sigma}^{\dagger}\left(c_{i \sigma}\right)$ is the usual fermionic creation (annihilation) operator. $\epsilon_{i}=\operatorname{diag}\left(\epsilon_{i \uparrow}, \epsilon_{i \downarrow}\right)$ where $\epsilon_{i \sigma}$ represents the site energy. In the presence of AAH modulation, the site energies are expressed as [71-73] $\epsilon_{i \uparrow}=\epsilon_{i \downarrow}=W \cos \left(2 \pi b i+\phi_{v}\right)$, where $W$ measures the strength of the cosine modulation, $b$ is an irrational number and $\phi_{v}$ is the AAH phase factor. In our calculations, we set $b=(1+\sqrt{5}) / 2$ without loss of any generality. With a suitable setup, one can regulate the phase factor $\phi_{v}$, and here we discuss its effect on SP. The term $\vec{h}_{i} \cdot \vec{\sigma}$ is responsible for spin dependent scattering [64-69], where $\vec{h}_{i}$ is the spin-flip scattering factor and $\vec{\sigma}$ denotes the Pauli spin vector. It is a well known scattering phenomenon and has been elaborately studied in literature (see Refs. [64-69], and the references therein). The strength $h_{i}$ is usually very large and in some cases it becomes an order of magnitude higher than the SO coupling [64]. Because of this fact, we get large mismatch between the two spin channels. The orientation of the spin flip vector is described by the conventional polar and azimuthal angles, $\theta_{i}$ and $\varphi_{i}$, respectively. Here, it is relevant to note that, in the present formulation we ignore the effect of interaction among the neighboring magnetic moments. It is well established that the moment-moment interaction can be expreesed as an effective Zeeman like term which represents the interaction of localized magnetic moments with an effective $B$-field (commonly referred as the "molecular field"). Compared to the interaction of itinerant electrons with local moments, as the Zeeman coupling is too weak, due to the existance of the factor $\mu_{B}$, no appreciable change is expected in SP even when the magnetic field is too high.

The rest part of $H_{m a g}$ is associated with the hopping of an electron from one site to its neighboring sites. $\tilde{t}=\operatorname{diag}(\tilde{t}, \tilde{t})$. In the presence of light irradiation, the nearest-neighbor hopping (NNH) strength $t$ gets renormalized and it takes the form $[59,60]$

$$
\begin{aligned}
\tilde{t} & =t \frac{1}{\mathcal{T}} \int_{0}^{\mathcal{T}} e^{j(p-q) \Omega \tau} e^{\vec{A} \cdot \vec{a}} d \tau \\
& =t J_{(p-q)}(\Lambda)
\end{aligned}
$$

where $J_{(p-q)}$ is the $(p-q)$ th order Bessel function of the first kind. 
To include the effect of irradiation we follow the Floquet ansatz. Within the minimal coupling scheme the irradiation can be simplified through a vector potential $\vec{A}(\tau)$ like $[59,60]$

$$
\vec{A}(\tau)=\left\{A_{x} \sin (\Omega \tau), A_{y} \sin (\Omega \tau+\phi)\right\}
$$

where $A_{x}$ and $A_{y}$ represent the amplitudes and $\phi$ corresponds to the phase. Depending on these parameters we get linear, elliptical and circularly polarized lights. The vector potential satisfies the relation $\vec{A}(\mathcal{T}+\tau)=\vec{A}(\tau)$ where $\mathcal{T}(=2 \pi / \Omega)$ is the time period of the driving field. $\vec{a}$ is the lattice vector and $j=\sqrt{-1}$. As we are working with a strictly 1D system, $\Lambda$ simplifies to $\Lambda=A_{x} a$, and, $A_{y}$ and $\phi$ do not have any explicit roles in our analysis.

The other three sub-Hamiltonians of Equation (1) can be written in a much simpler way as they do not include any kind of magnetic interaction and irradiation. They are expressed as:

$$
\begin{gathered}
H_{S}=\sum_{i \leq-1} a_{i}^{\dagger} \epsilon_{0} a_{i}+\sum_{i \leq-1}\left(a_{i+1}^{\dagger} t_{0} a_{i}+\text { h.c. }\right) \\
H_{D}=\sum_{i \geq N+1} b_{i}^{\dagger} \epsilon_{0} b_{i}+\sum_{i \geq N+1}\left(b_{i+1}^{\dagger} t_{0} b_{i}+\text { h.c. }\right) \\
H_{\text {tun }}=c_{1}^{\dagger} t_{S} a_{-1}+c_{N}^{\dagger} t_{D} b_{N+1}+\text { h.c. }
\end{gathered}
$$

where $a_{i}$ and $b_{i}$ are of the similar form like $c_{i}$, and they contain usual fermionic creation and annihilation operators. The electrodes $S$ and $D$ are parametrized by on-site energy $\epsilon_{0}$ and $t_{0}$, respectively. The parameters $t_{S}$ and $t_{D}$ represent the coupling strengths of $S$ and $\mathrm{D}$ with the magnetic chain, respectively.

\subsection{Theoretical Formulation}

The spin polarization coefficient of the above Hamiltonian (Equation (1)) is obtained by determining the spin dependent transmission probabilities which we compute by using Green's function formalism where the effects of $\mathrm{S}$ and $\mathrm{D}$ are incorporated through selfenergy corrections. For comprehensive analysis of this formalism, we recommend the general readers to see the Refs. [78,79]. The effective Green's function of the magnetic chain reads as $[78,79]$

$$
G^{r}=\left(G^{a}\right)^{\dagger}=\left(E I-H_{m a g}-\Sigma_{S}-\Sigma_{D}\right)^{-1}
$$

where $E$ is the energy of the incoming electron from the source end and $I$ is the identity matrix having dimension $(2 N \times 2 N) . \Sigma_{S}$ and $\Sigma_{D}$ are the self-energy matrices of the $S$ and $\mathrm{D}$, respectively. From this Green's function, we determine the transmission probabilities using the Fisher-Lee expression [78-82]

$$
T_{\sigma \sigma^{\prime}}=\operatorname{Tr}\left[\Gamma_{S}^{\sigma} G^{r} \Gamma_{D}^{\sigma^{\prime}} G^{a}\right]
$$

where $\Gamma_{S}^{\sigma}$ and $\Gamma_{D}^{\sigma^{\prime}}$ are the coupling matrices. These coupling matrices are found from the self-energy matrices via the relations

$$
\Gamma_{S(D)}^{\sigma\left(\sigma^{\prime}\right)}=i\left[\Sigma_{S(D)}^{\sigma\left(\sigma^{\prime}\right)}-\left(\Sigma_{S(D)}^{\sigma\left(\sigma^{\prime}\right)}\right)^{\dagger}\right]
$$

Using Equation (9) we get both pure $\left(\sigma=\sigma^{\prime}\right)$ and spin flip $\left(\sigma \neq \sigma^{\prime}\right)$ transmissions through the magnetic junction. With these co-efficients we get the net up and down spin transmission probabilities as

$$
\begin{aligned}
& T_{\uparrow}=T_{\uparrow \uparrow}+T_{\downarrow \uparrow}, \\
& T_{\downarrow}=T_{\downarrow \downarrow}+T_{\uparrow \downarrow} .
\end{aligned}
$$


Determining $T_{\uparrow}$ and $T_{\downarrow}$, we evaluate the spin polarization coefficient $P$ with the relation [83-87]

$$
P=\frac{T_{\uparrow}-T_{\downarrow}}{T_{\uparrow}+T_{\downarrow}} .
$$

$P= \pm 1$ represents complete up (down) spin polarization, while $P=0$ denotes vanishing polarization.

\section{Numerical Results and Discussion}

Based on the above theoretical framework now we present the results. Our primary goal is to achieve a high degree of spin polarization and tuning its phase by means of irradiation. All the results are worked out in the high frequency limit which is defined as $\hbar \Omega>>t$, and in this limiting condition only the lowest order Floquet band, i.e., $p=q=0$ contributes. For the chosen parameter values, the frequency becomes $\Omega \geq 10^{15} \mathrm{~Hz}$ which denotes the far-infrared (FIR) region. The intensity of the irradiation is of the order of $10^{7} \mathrm{~W} / \mathrm{m}^{2}$ which is within the experimental limit. The corresponding electric $(\mathcal{E})$ and magnetic $(B=\mathcal{E} / c)$ fields are $10^{5} \mathrm{~V} / \mathrm{m}$ and $10^{-4}$ Tesla, respectively.

The other common set of parameter values that we choose for our calculations are as follows. In the electrodes $S$ and $D$ we take $\epsilon_{0}=0$ and $t_{0}=2 \mathrm{eV}$, and they are coupled to the magnetic chain via the coupling strengths $t_{S}=t_{D}=1 \mathrm{eV}$. In the magnetic chain we choose $t=1 \mathrm{eV}$, AAH modulation strength $W=1 \mathrm{eV}$, spin flip parameter $h_{i}=1 \mathrm{eV} \forall i$, $\varphi_{i}=0 \forall i$. Unless specified, we fix the total number of sites in the magnetic chain $N=30$. The parameter values those are not constant are given in the appropriate places, and all the other energies are also measured in unit of eV. Throughout the calculations we restrict ourselves in the zero temperature limit. This is a realistic approximation as long as the average energy level spacing is higher than the thermal energy, and for small scale systems (even for $N<200$ ) this condition can be easily achieved. It is also important to note that one cannot increase the chain length as much as it is possible, since we need to confine the system size within the spin coherence length. Otherwise no such phenomenon will be observed.

Let us begin with Figure 2 where spin dependent transmission probabilities $T_{\sigma}$ along with spin polarization co-efficient $P$ are shown as a function of energy $E$ for some specific values of $A_{x}$. Several key features are emerged those are analyzed as follows. At a first glance we see that the transmission spectrum is highly fragmented and gapped in nature. This is solely due to the cosine modulation in site energies, as gapped energy spectrum is the generic feature of an AAH system. The transmission spectrum is a direct manifestation of the energy values. Unlike the perfect magnetic chain where energy spectrum is not gapped, for the AAH case we have a finite probability to get non-zero spin polarization at different energy zones. More importantly, even near the energy band centre we can get a reasonably large spin polarization. This feature is always desirable since one can easily place the Fermi level close to the central region, apart from placing it near the energy band edges. The role of irradiation is of course fascinating. For $A_{x}=0$, a finite overlap between up and down spin transmission probabilities takes place, following the up and down spin energy channels, for a broad energy region. Therefore, for these energy zones spin polarization becomes too small. Whereas, the transmission spectra start to get shifted with $A_{x}$ and they are almost separated for higher $A_{x}$, which is clearly visible by comparing the spectra given in the left column of Figure 2.

The shifting of transmission probabilities with $A_{x}$ is entirely due to the modification of energy eigenvalues of the magnetic chain, since the NNH strength gets renormalized in presence of the irradiation (see Equation (3)). As effective hopping decreases compared to the irradiation free case, we get reduced allowed energy windows for up and down spin electrons, and thus the transmission spectra. All the characteristic features are directly reflected in the $P$ - $E$ spectra (see right column of Figure 2). Almost $100 \%$ spin polarization can be achieved for the entire allowed energy zones by suitably adjusting the irradiation 
parameter. It gives a clear signature of achieving externally controlled spin polarization through a magnetic nanojunction.
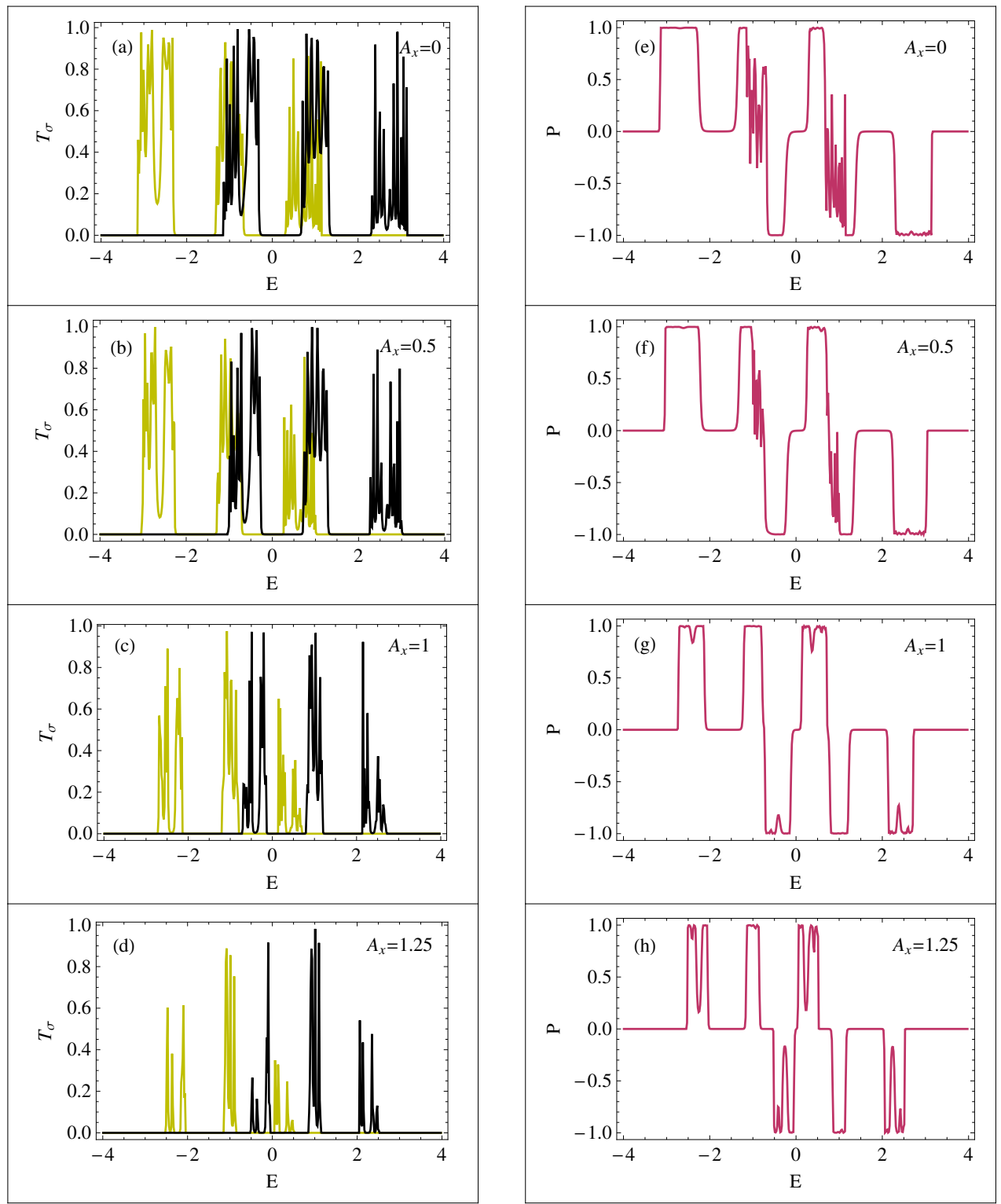

Figure 2. Up and down spin transmission probabilities ( $T_{\uparrow} \rightarrow$ light green color and $T_{\downarrow} \rightarrow$ black color) and spin polarization coefficient $P$ as a function of energy $E$ at four typical values of $A_{x}$. Here we set $\theta_{i}=0 \forall i$ and AAH phase $\phi_{v}=0$.

The above analysis gives rise to a very fundamental question that how the spin polarization can vary if we tune $A_{x}$ continuously, instead of fixing it at some typical values. To demonstrate it, in Figure 3 we show the dependence of $P$ as a function of $A_{x}$ by varying it in a wide range. The results are presented for two distinct energies, $E=-1 \mathrm{eV}$ and $1 \mathrm{eV}$. Both for these two energies, $P$ shows a large oscillation with increasing amplitude for lower $A_{x}$, and eventually saturates exhibiting $100 \%$ polarization for higher $A_{x}$. At $E=-1 \mathrm{eV}$ or $1 \mathrm{eV}$, there is a finite overlap between the two transmission functions when $A_{x}=0$ and thus $P$ becomes very small. The spectral properties and thus the transmission spectra get modified with the alteration of $A_{x}$. However, the fact is that the NNH strength $t$ does not monotonically decrease with $A_{x}$ as it follows the zeroth order Bessel function of the first kind (see Equation (3)). Due to this reason, in some cases we get finite overlap between $T_{\uparrow}$ and $T_{\downarrow}$ which yields lesser $P$. On the other hand when the overlap is less, higher $P$ is 
achieved. This is the underlying mechanism to have oscillatory behavior of $P$ with $A_{x}$. For large $A_{x}$, when $T_{\uparrow}$ and $T_{\downarrow}$ are practically separated we get the maximum SP. Depending on the dominating factor among $T_{\uparrow}$ and $T_{\downarrow}$, we get either $100 \%$ up or down spin polarization. These results clearly justify that the degree of SP can be tuned in a wide range by regulating $A_{x}$, without altering any other physical parameters describing the system.

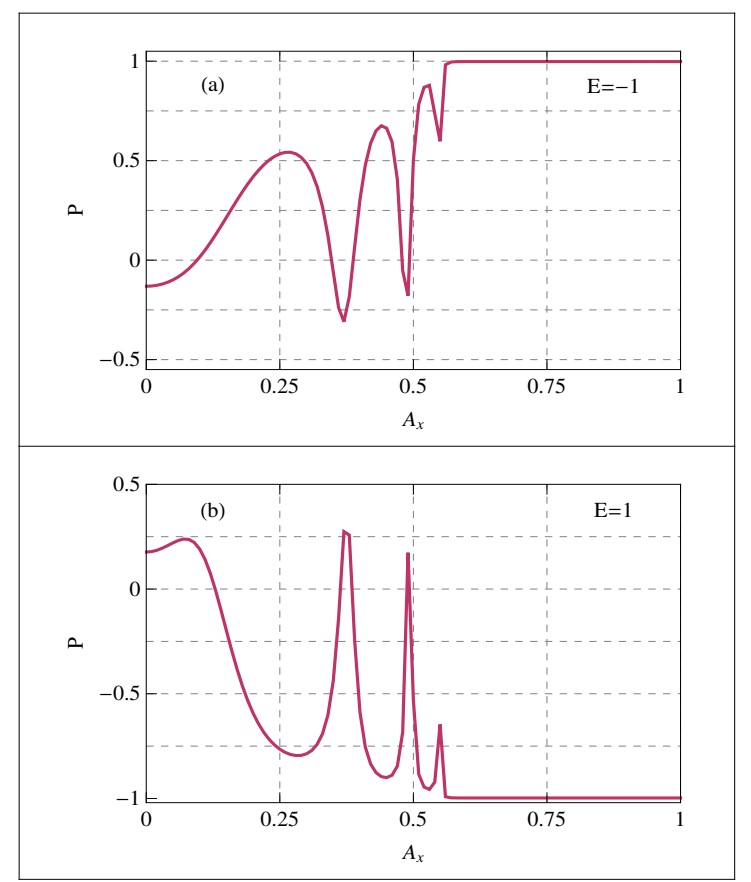

Figure 3. $P-A_{x}$ characteristics at two distinct energies. All the other physical parameters kept unchanged as taken in Figure 2.

The results discussed so far are computed for the magnetic chain where all the magnetic moments are aligned along $+Z$ direction i.e., $\theta=0$ (we refer $\theta_{i}=\theta$ as we assume that all the moments are aligned in a particular direction). For $\theta=0$, there is no spin flipping i.e., $T_{\uparrow \downarrow}=T_{\downarrow \uparrow}=0$, since in this case $\sigma_{x}$ and $\sigma_{y}$ do not involve into the TB Hamiltonian Equation (2) and it becomes exactly diagonal. However, finite spin flipping occurs as long as the moments are aligned in a particular direction with respect to $+Z$ axis, and to reveal the $\theta$ dependence on SP, in Figure 4 , we present $P-\theta$ characteristics by changing $\theta$ in a wide range, for two different values of $A_{x}$, considering the identical energy values as taken in Figure 3. Both for the orange and black curves, associated with $A_{x}=0.5$ and 1 respectively, the spin polarization co-efficient shows a complete phase reversal under rotating the magnetic moments. For the two typical values of $\theta$, SP drops exactly to zero, as expected. With the change of $\theta$ spectral behavior gets changed and hence the SP. Thus, the alignment of the magnetic moments has an important role in SP.

To explore the explicit dependance of SP on both $\theta$ and $A_{x}$, in Figure 5, we present a density plot of $P$ by varying these factors in a broad range fixing the energies at some specific values. From these spectra we get a clear hint about the range of physical parameters for which large degree of spin polarization can be obtained for this magnetic junction. The phase reversal is also clearly noticed.

Now we focus our attention to examine the critical role played by the AAH phase $\phi_{v}$ on spin selective electron transfer. In Figure 6, we present the variation of spin polarization coefficient as a function of $\phi_{v}$ for two distinct energies. The orange curve is for $E=-0.75 \mathrm{eV}$, while the other one is for $E=0.75 \mathrm{eV}$. A reasonably large change is reflected from both these two curves. Regulating $\phi_{v}$, that can be done externally, we can change the available energy channels between the electrodes as the effective site energies of the magnetic chain are modified, and therefore, the degree of spin polarization can be tuned. Thus, along with 
the irradiation parameter, the AAH phase can also be considered as a suitable parameter for regulating the spin transfer.

To have a more clear picture and to understand precisely the interplay between the light parameter and the AAH phase, in Figure 7, we present a density plot of SP by varying simultaneously $A_{x}$ and $\phi_{v}$ in a broad range. Some typical energies are selected like what we consider in Figure 5. Quite interestingly we find that, the degree of SP and its sign can be monitored selectively by adjusting $A_{x}$ and $\phi_{v}$. The phenomenon persists for a wide range of these parameters which suggests that extremely fine tuning is no longer required. It certainly gives us a confidence that the present findings can be tested in a suitable laboratory setup.

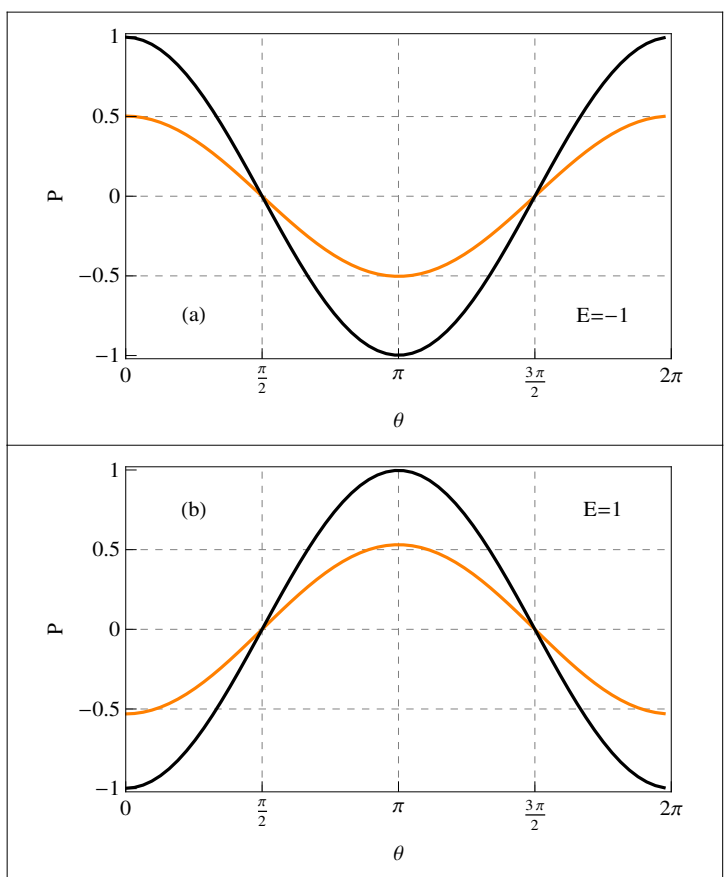

Figure 4. $P$ as a function of $\theta\left(\theta_{i}=\theta \forall i\right)$ at two different energies, where the orange and black lines correspond to $A_{x}=0.5$ and 1 , respectively. The AAH phase $\phi_{v}=0$.

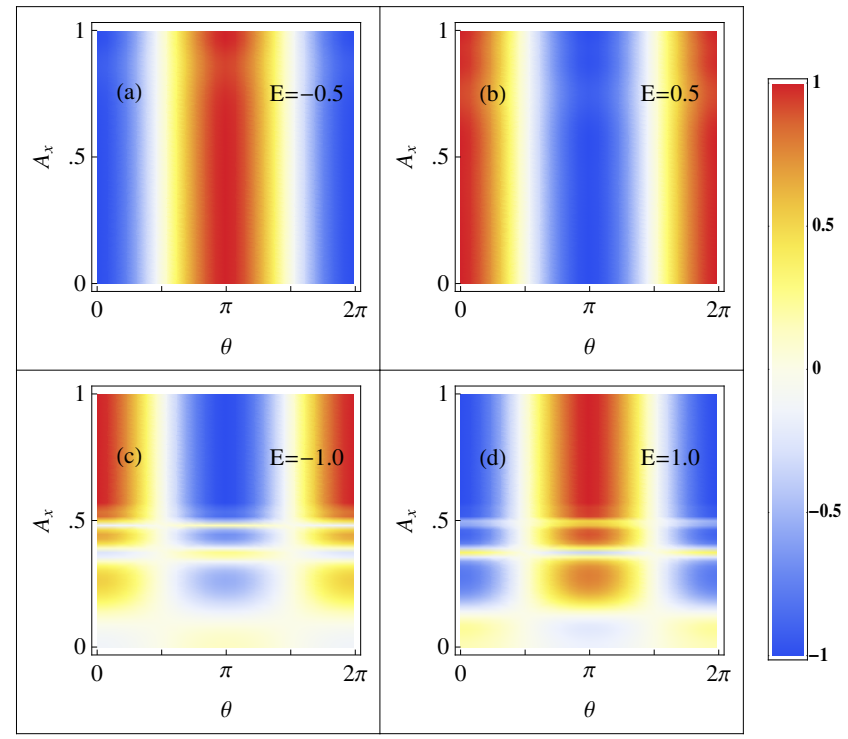

Figure 5. Simultaneous variation (density plot) of $P$ with $\theta\left(\theta_{i}=\theta \forall i\right)$ and $A_{x}$ at some specific values of energy. The AAH phase $\phi_{v}=0$. 


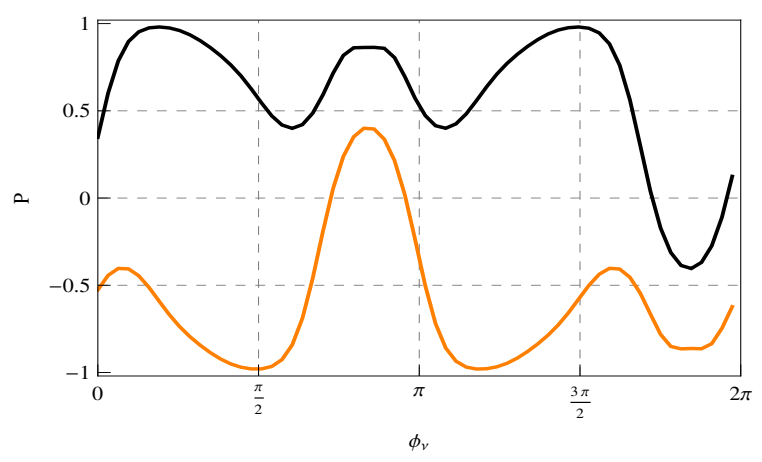

Figure 6. Spin polarization co-efficient $P$ as a function of $\phi_{v}$ at two typical energies, where the orange and black curves are for $E=-0.75 \mathrm{eV}$ and $E=0.75 \mathrm{eV}$, respectively. Here, we consider $A_{x}=0.75$ and $\theta_{i}=0 \forall i$.

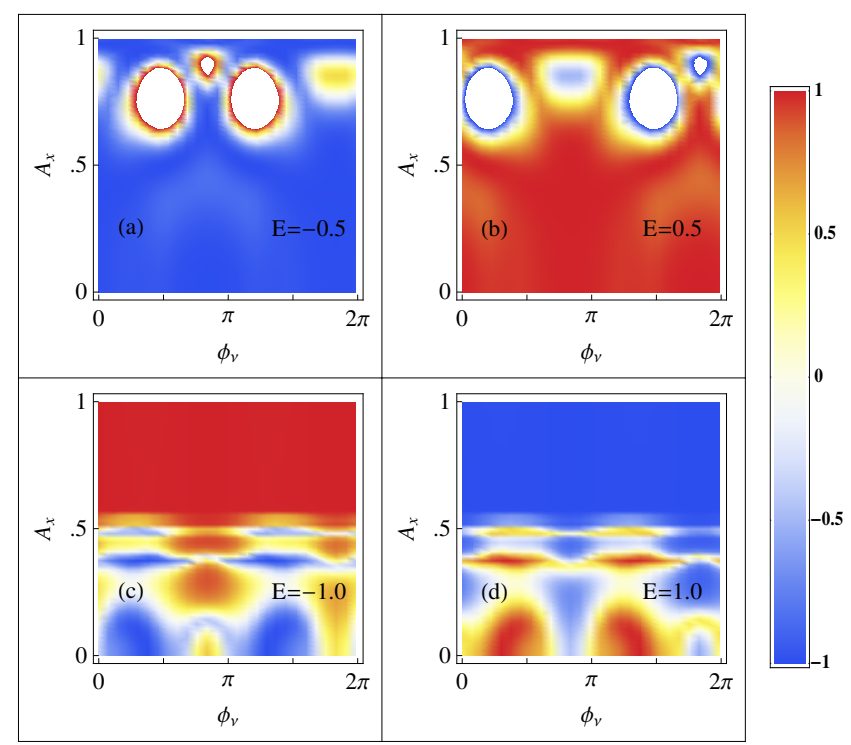

Figure 7. Simultaneous variation (density plot) of $P$ with AAH phase $\phi_{v}$ and light parameter $A_{x}$ at some specific energies. Here we set $\theta_{i}=0 \forall i$.

Finally, keeping in mind the possible experimental realization of our prescription it is indeed required to investigate the effect of system size on spin dependent transmission probabilities and the spin polarization co-efficient. To explore it, in Figure 8, we show the dependence of these physical quantities on system size $N$ by varying it in a wide range at two typical energies. For both of these energies, $T_{\sigma}$ and $P$ exhibit large amplitude oscillation with $N$. This is solely due to the effect of quantum interference among electronic waves, and can be observed for other energies as well, which we confirm through our detailed numerics. The crucial thing is that the oscillating nature persists even for a reasonably large system size, and therefore, we can safely verify our proposal in a suitable laboratory setup. In this context it is relevant to note that similar kind of oscillation in transport quantities by varying system size has also been reported in different contemporary works, and for the ordered systems it can be tested even analytically $[65,88,89]$. 

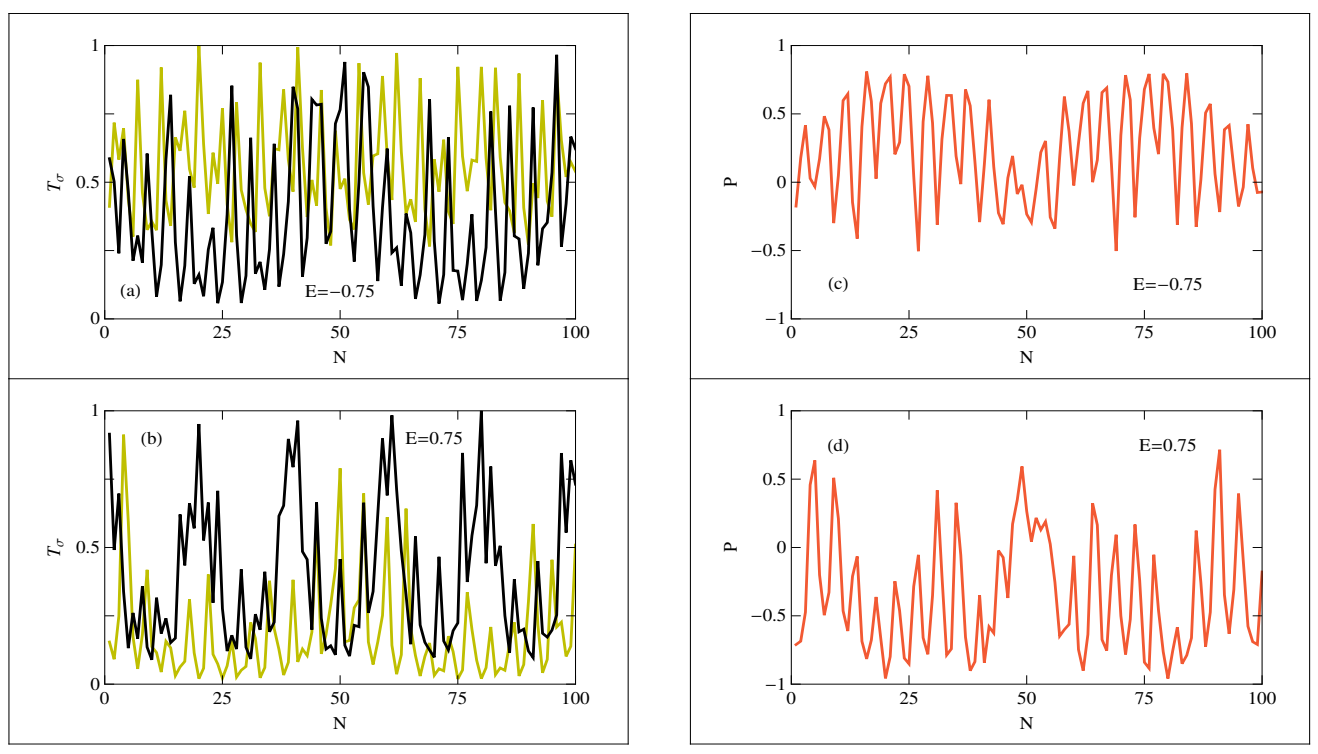

Figure 8. Spin dependent transmission probabilities ( $T_{\uparrow} \rightarrow$ light green color and $T_{\uparrow} \rightarrow$ black color) and corresponding spin polarization co-efficient $P$ as a function of system size $N$ at two typical energies. Here we set $A_{x}=0.25, \phi_{v}=0$ and $\theta_{i}=0 \forall i$.

\section{Closing Remarks}

A possible route of engineering spin polarization by means of light irradiation is proposed considering a tight-binding magnetic chain with cosine modulation in site energies. Each site of the magnetic system possesses a finite magnetic moment which interacts with the itinerent electrons. Because of this interaction, up and down spin channels get misaligned. Simulating the nanojunction, formed by placing the magnetic chain between two non-magnetic contacts, within a tight-binding framework, we determine spin dependent transmission probabilities using the Green's function formalism. From the transmission function we evaluate spin polarization co-efficient. The interplay between the irradiation, included into the Hamiltonian following the standard Floquet prescription, and the AAH potential has an important role and we investigate it critically on SP. Apart from achieving a high degree of spin polarization we can also selectively tune its phase with the help of irradiation. The peculiar gapped nature of up and down spin energy channels in presence of cosine modulation allows us to get higher filtration efficiency at multiple energy zones, and most importantly, it is also possible near the energy band centre together with other energy regions. Our analysis can be utilized to investigate spin dependent transport phenomena in different driven magnetic systems with correlated impurities.

Author Contributions: This paper was accomplished based on a collaborative work of the authors. S.K.M. conceived the project. A.K. and S.K.M. performed numerical calculations. All the authors have analyzed the data and co-wrote the paper. All authors have read and agreed to the published version of the manuscript.

Funding: This research was funded by FONDECYT 1180905 and Centers of Excellence with BASAL/ ANID financing, Grant AFB180001, CEDENNA.

Institutional Review Board Statement: Not applicable.

Informed Consent Statement: Not applicable.

Data Availability Statement: The data that support the findings of this study are available from the corresponding author upon reasonable request.

Acknowledgments: AK thanks DST-SERB, Government of India (Project No. EMR/2017/000504). JHOS recognizes Universidad Pedagógica y Tecnológica de Colombia (Project No. SGI 2834). 
Conflicts of Interest: The authors declare no conflict of interest.

\section{References}

1. Wolf, S.A.; Awschalom, D.D.; Buhrman, R.A.; Daughton, J.M.; von Molnár, S.; Roukes, M.L.; Chtchelkanova, A.Y.; Trege, D.M. Spintronics: A spin-based electronics vision for the future. Science 2001, 294, 1488. [CrossRef]

2. Zutić, I.; Fabian, J.; Sarma, S.D. Spintronics: Fundamentals and applications. Rev. Mod. Phys. 2004, 76, 323.

3. Sahoo, S.; Kontos, T.; Furer, J.; Hoffmann, C.; Gräber, M.; Cottet, A.; Schönenberger, C. Electric field control of spin transport. Nat. Phys. 2005, 1, 99. [CrossRef]

4. Datta, S.; Das, B. Electronic analog of the electro-optic modulator. Appl. Phys. Lett. 1990, 56, 665. [CrossRef]

5. Tsukagoshi, K.; Alphenaar, B.W.; Ago, H. Coherent transport of electron spin in a ferromagnetically contacted carbon nanotube. Nature 1999, 401, 572. [CrossRef]

6. Kobayashi, T.; Nakata, Y.; Yaji, K.; Shishidou, T.; Agterberg, D.; Yoshizawa, S.; Komori, F.; Shin, S.; Weinert, M.; Uchihashi, T.; et al. Orbital Angular Momentum Induced Spin Polarization of 2D Metallic Bands. Phys. Rev. Lett. 2020, 125, 176401. [CrossRef]

7. Yuan, L.-D.; Wang, Z.; Luo, J.-W.; Rashba, E.I.; Zunger, A. Giant momentum-dependent spin splitting in centrosymmetric low-Z antiferromagnets. Phys. Rev. B 2020, 102, 014422. [CrossRef]

8. Manchon, A.; Zelezny, J.; Miron, I.M.; Jungwirth, T.; Sinova, J.; Thiaville, A.; Garello, K.; Gambardella, P. Current-induced spin-orbit torques in ferromagnetic and antiferromagnetic systems. Rev. Mod. Phys. 2019, 91, 035004. [CrossRef]

9. Jiao, Y.; Ma, F.; Zhang, C.; Bell, J.; Sanvito, S.; Du, A. First-Principles Prediction of Spin-Polarized Multiple Dirac Rings in Manganese Fluoride. Phys. Rev. Lett. 2017, 119, 016403. [CrossRef]

10. Han, W.; Maekawa, S.; Xie, X.-C. Spin current as a probe of quantum materials. Nat. Mat. 2020, 19, 139. [CrossRef] [PubMed]

11. Prinz, G.A. Magnetoelectronics. Science 1998, 282, 1660.

12. Schmidt, G.; Ferrand, D.; Molenkamp, L.W.; Filip, A.T.; van Wees, B.J. Fundamental obstacle for electrical spin injection from a ferromagnetic metal into a diffusive semiconductor. Phys. Rev. B 2000, 62, R4790. [CrossRef]

13. Wu, M.W.; Zhou, J.; Shi, Q.W. Spin-dependent quantum transport in periodic magnetic modulations: Aharonov-Bohm ring structure as a spin filter. Appl. Phys. Lett. 2004, 85, 1012. [CrossRef]

14. Kiselev, A.A.; Kim, K.W. T-shaped ballistic spin filter. Appl. Phys. Lett. 2001, 78, 775. [CrossRef]

15. Governale, M.; Boese, D.; Zülicke, U.; Schroll, C. Filtering spin with tunnel-coupled electron wave guides. Phys. Rev. B 2002, 65, 140403. [CrossRef]

16. Maiti, S.K. Curvature effect on spin polarization in a three-terminal geometry in presence of Rashba spin-orbit interaction. Phys. Lett. A 2015, 379, 361. [CrossRef]

17. Khodas, M.; Shekhter, A.; Finkel'stein, A.M. Spin Polarization of Electrons by Nonmagnetic Heterostructures: The Basics of Spin Optics. Phys. Rev. Lett. 2004, 92, 086602. [CrossRef] [PubMed]

18. Streda, P.; Seba, P. Antisymmetric Spin Filtering in One-Dimensional Electron Systems with Uniform Spin-Orbit Coupling. Phys. Rev. Lett. 2003, 90, 256601. [CrossRef]

19. Dey, M.; Maiti, S.K.; Karmakar, S.N. Magnetic quantum wire as a spin filter: An exact study. Phys. Lett. A $2010,374,1522$. [CrossRef]

20. Dey, M.; Maiti, S.K.; Karmakar, S.N. Spin transport through a quantum network: Effects of Rashba spin-orbit interaction and Aharonov-Bohm flux. J. Appl. Phys. 2011, 109, 024304. [CrossRef]

21. Winkler, R. Spin-Orbit Coupling Effects in Two-Dimensional Electron and Hole Systems; Springer Tracts in Modern Physics; Springer: New York, NY, USA, 2003; Volume 191.

22. Pareek, T.P. Pure Spin Currents and the Associated Electrical Voltage. Phys. Rev. Lett. 2004, 92, 076601. [CrossRef]

23. Sun, Q.F.; Xie, X.C. Bias-controllable intrinsic spin polarization in a quantum dot: Proposed scheme based on spin-orbit interaction. Phys. Rev. B 2006, 73, 235301. [CrossRef]

24. Maiti, S.K.; Dey, M.; Sil, S.; Chakrabarti, A.; Karmakar, S.N. Magneto-transport in a mesoscopic ring with Rashba and Dresselhaus spin-orbit interactions. Europhys. Lett. 2011, 95, 57008. [CrossRef]

25. Chi, F.; Zheng, J.; Sun, L.L. Spin-polarized current and spin accumulation in a three-terminal two quantum dots ring. Appl. Phys. Lett. 2008, 92, 172104. [CrossRef]

26. Maiti, S.K. Determination of Rashba and Dresselhaus spin-orbit fields. J. Appl. Phys. 2011, 110, 064306. [CrossRef]

27. Sil, S.; Maiti, S.K.; Chakrabarti, A. Interplay of magnetic field and geometry in magneto-transport of mesoscopic loops with Rashba and Dresselhaus spin-orbit interactions. J. Appl. Phys. 2012, 112, 024321. [CrossRef]

28. Maiti, S.K.; Sil, S.; Chakrabarti, A. A proposal for the measurement of Rashba and Dresselhaus spin-orbit interaction strengths in a single sample. Phys. Lett. A 2012, 376, 2147. [CrossRef]

29. Yin, H.-T.; Lu, T.-Q.; Liu, X.-J.; Xue, H.-J. Voltage-controllable spin-polarized transport through parallel-coupled double quantum dots with Rashba spin-orbit interaction. Phys. Lett. A 2009, 373, 285. [CrossRef]

30. Ganguly, S.; Basu, S.; Maiti, S.K. Unconventional charge and spin dependent transport properties of a graphene nanoribbon with line-disorder. Europhys. Lett. 2018, 124, 57003. [CrossRef]

31. Patra, M.; Maiti, S.K. Unconventional low-field magnetic response of a diffusive ring with spin-orbit coupling. Phys. Lett. A 2017, 381, 221. [CrossRef]

32. Bychkov, Y.A.; Rashba, E.I. Properties of a 2D electron gas with lifted spectral degeneracy. Sov. Phys. JETP Lett. 1984, $39,78$. 
33. Dresselhaus, G. Spin-Orbit Coupling Effects in Zinc Blende Structures. Phys. Rev. 1955, 100, 580. [CrossRef]

34. Földi, O. Kálmán, P.; Benedict, M.G.; Peeters, F.M. Quantum rings as electron spin beam splitters. Phys. Rev. B 2006, 73, 155325. [CrossRef]

35. Hatano, N.; Shirasaki, R.; Nakamura, H. Non-Abelian gauge field theory of the spin-orbit interaction and a perfect spin filter. Phys. Rev. A 2007, 75, 032107. [CrossRef]

36. Dey, M.; Maiti, S.K.; Karmakar, S.N. Spin Hall effect in a Kagome lattice driven by Rashba spin-orbit interaction. J. Appl. Phys. 2012, 112, 024322. [CrossRef]

37. Földi, O. Kálmán, P.; Benedict, M.G.; Peeters, F.M. Networks of Quantum Nanorings: Programmable Spintronic Devices. Nano Lett. 2008, 8, 2556. [CrossRef]

38. Dey, M.; Maiti, S.K.; Sil, S.; Karmakar, S.N. Spin-orbit interaction induced spin selective transmission through a multi-terminal mesoscopic ring. J. Appl. Phys. 2013, 114, 164318. [CrossRef]

39. Cohen, G.; Hod, O.; Rabani, E. Constructing spin interference devices from nanometric rings. Phys. Rev. B 2007, 76, 235120. [CrossRef]

40. Maiti, S.K. Externally controlled selective spin transfer through a two-terminal bridge setup. Eur. Phys. J. B 2015, 88, 172. [CrossRef]

41. Földi, O.; Kálmán, P.; Peeters, F.M. Stability of spintronic devices based on quantum ring networks. Phys. Rev. B 2009, 80, 125324. [CrossRef]

42. Patra, M.; Maiti, S.K. Externally controlled high degree of spin polarization and spin inversion in a conducting junction: Two new approaches. Sci. Rep. 2017, 7, 14313. [CrossRef]

43. Göhler, B.; Hamelbeck, V.; Markus, T.Z.; Kettner, M.; Hanne, G.F.; Vager, Z.; Naaman, R.; Zacharias, H. Spin Selectivity in Electron Transmission Through Self-Assembled Monolayers of Double-Stranded DNA. Science 2011, 331, 894. [CrossRef] [PubMed]

44. Ganguly, S.; Basu, S.; Maiti, S.K. Interface sensitivity on spin transport through a three-terminal graphene nanoribbon. Superlattices Microstruct. 2018, 120, 650. [CrossRef]

45. Guo, A.-M.; Sun, Q.-F. Spin-Selective Transport of Electrons in DNA Double Helix. Phys. Rev. Lett. 2012, 108, 218102. [CrossRef]

46. Ganguly, S.; Basu, S.; Maiti, S.K. Controlled engineering of spin polarized transport properties in a zigzag graphene nanojunction. Europhys. Lett. 2018, 124, 17005. [CrossRef]

47. Guo, A.-M.; Sun, Q.-F. Spin-dependent electron transport in protein-like single-helical molecules. Proc. Natl. Acad. Sci. USA 2014, 111, 11658. [CrossRef] [PubMed]

48. Kuemmeth, F.; Ilani, S.; Ralph, D.C.; McEuen, P.L. Coupling of spin and orbital motion of electrons in carbon nanotubes. Nature 2008, 452, 448. [CrossRef] [PubMed]

49. Long, W.; Sun, Q.F.; Guo, H.; Wang, J. Gate-controllable spin battery. Appl. Phys. Lett. 2003, 83, 1397. [CrossRef]

50. Zhang, P.; Xue, Q.K.; Xie, X.C. Spin Current through a Quantum Dot in the Presence of an Oscillating Magnetic Field. Phys. Rev. Lett. 2003, 91, 196602. [CrossRef]

51. Adachi, H.; Ino, H. A ferromagnet having no net magnetic moment. Nature 1999, 401, 148. [CrossRef]

52. Xu, Y.; Wang, S.; Xia, K. Spin-Transfer Torques in Antiferromagnetic Metals from First Principles. Phys. Rev. Lett. 2008, 100, 226602. [CrossRef]

53. Jungwirth, T.; Sinova, J.; Matri, X.; Wunderlich, J.; Felser, C. The multiple directions of antiferromagnetic spintronics. Nat. Phys. 2018, 14, 200. [CrossRef]

54. Jungfleisch, M.B.; Zhang, W.; Hoffmann, A. Perspectives of antiferromagnetic spintronics. Phys. Lett. A 2018, 13, 382. [CrossRef]

55. Duine, R.A.; Lee, K.; Parkin, S.P.; Stiles, M.D. Synthetic antiferromagnetic spintronics. Nat. Phys. 2018, 14, 217. [CrossRef] [PubMed]

56. Gupta, D.D.; Maiti, S.K. Can a sample having zero net magnetization produce polarized spin current? J. Phys. Condens. Matter 2020, 32, 505803. [CrossRef]

57. Grifoni, M.; Hänggi, P. Driven quantum tunneling. Phys. Rep. 1998, 304, 229. [CrossRef]

58. Kohler, S.; Lehmann, J.; Hänggi, P. Driven quantum transport on the nanoscale. Phys. Rep. 2005, 406, 379. [CrossRef]

59. Delplace, P.; Gómez-Lexoxn, A.; Platero, G. Merging of Dirac points and Floquet topological transitions in ac-driven graphene. Phys. Rev. B 2013, 88, 245422. [CrossRef]

60. Gómez-Lexoxn, A.; Platero, G. Floquet-Bloch Theory and Topology in Periodically Driven Lattices. Phys. Rev. Lett. 2013, 110, 200403. [CrossRef] [PubMed]

61. Eckardt, A.; Anisimovas, E. High-frequency approximation for periodically driven quantum systems from a Floquet-space perspective. New. J. Phys. 2015, 17, 093039. [CrossRef]

62. Goldman, N.; Dalibard, J.; Aidelsburger, M.; Cooper, N.R. Periodically driven quantum matter: The case of resonant modulations. Phys. Rev. A 2015, 91, 033632. [CrossRef]

63. Sarkar, M.; Dey, M.; Maiti, S.K.; Sil, S. Engineering spin polarization in a driven multistranded magnetic quantum network. Phys. Rev. B 2020, 102, 195435. [CrossRef]

64. Su, Y.-H.; Chen, S.-H.; Hu, C.D.; Chang, C.-R. Competition between spin-orbit interaction and exchange coupling within a honeycomb lattice ribbon. J. Phys. D Appl. Phys. 2016, 49, 015305. [CrossRef]

65. Sarkar, S.; Maiti, S.K. Spin-selective transmission through a single-stranded magnetic helix. Phys. Rev. B 2019, 100, 205402. [CrossRef] 
66. Shokri, A.A.; Mardaani, M.; Esfarjani, K. Spin filtering and spin diode devices in quantum wire systems. Physica E 2005, 27, 325. [CrossRef]

67. Shokri, A.A.; Mardaani, M. Spin-flip effect on electrical transport in magnetic quantum wire systems. Solid State Commun. 2006, 137, 53. [CrossRef]

68. Peters, R.; Kawakami, N. Ferromagnetic state in the one-dimensional Kondo lattice model. Phys. Rev. B 2012, 86, 165107. [CrossRef]

69. Peters, R.; Kawakami, N.; Pruschke, T. Spin-Selective Kondo Insulator: Cooperation of Ferromagnetism and the Kondo Effect. Phys. Rev. Lett. 2012, 108, 086402. [CrossRef]

70. Aubry, S.; André, G. Analyticity breaking and Anderson localization in incommensurate lattices. Ann. Isr. Phys. Soc. 1980, 3, 133.

71. Kraus, Y.E.; Lahini, Y.; Ringel, Z.; Verbin, M.; Zilberberg, O. Topological States and Adiabatic Pumping in Quasicrystals. Phys. Rev. Lett. 2012, 109, 106402. [CrossRef] [PubMed]

72. Rossignolo, M.; Dell'Anna, L. Localization transitions and mobility edges in coupled Aubry-André chains. Phys. Rev. B 2019, 99, 054211. [CrossRef]

73. Sil, S.; Maiti, S.K.; Chakrabarti, A. Metal-insulator transition in an aperiodic ladder network: An exact result. Phys. Rev. Lett. 2008, 101, 076803. [CrossRef] [PubMed]

74. Roy, S.; Maiti, S.K. Tight-binding quantum network with cosine modulations: Electronic localization and delocalization. Eur. Phys. J. B 2019, 92, 267. [CrossRef]

75. Saha, S.; Maiti, S.K.; Karmakar, S.N. Multiple mobility edges in a 1D Aubry chain with Hubbard interaction in presence of electric field: Controlled electron transport. Physica E 2016, 83, 358. [CrossRef]

76. Maiti, S.K.; Sil, S.; Chakrabarti, A. Phase controlled metal-insulator transition in multi-leg quasiperiodic optical lattices. Ann. Phys. (N. Y.) 2017, 382, 150. [CrossRef]

77. Patra, M.; Maiti, S.K. Controlled charge and spin current rectifications in a spin polarized device. J. Magn. Magn. Mater. 2019, 484, 408. [CrossRef]

78. Datta, S. Electronic Transport in Mesoscopic Systems; Cambridge University Press: Cambridge, UK, 1997.

79. Datta, S. Quantum Transport: Atom to Transistor; Cambridge University Press: Cambridge, UK, 2005.

80. Fisher, D.S.; Lee, P.A. Relation between conductivity and transmission matrix. Phys. Rev. B 1981, 23, 6851. [CrossRef]

81. Ventra, M.D. Electrical Transport in Nanoscale Systems; Cambridge University Press: Cambridge, UK, 2008.

82. Nikolić, B.K.; Allen, P.B. Quantum transport in ballistic conductors: Evolution from conductance quantization to resonant tunnelling. J. Phys. Condens. Matter 2000, 12, 9629. [CrossRef]

83. Prinz, G.A. Spin-Polarized Transport. Phys. Today 1995, 48, 58. [CrossRef]

84. Jedema, F.F.; Filip, A.T.; van Wees, B.J. Electrical spin injection and accumulation at room temperature in an all-metal mesoscopic spin valve. Nature 2001, 410,345. [CrossRef]

85. Maekawa, S.; Shinjo, T. (Eds.) Spin Dependent Transport in Magnetic Nanostructures; Taylor \& Francis: London, UK, 2002.

86. Nikolić, B.K.; Souma, S. Decoherence of transported spin in multichannel spin-orbit-coupled spintronic devices: Scattering approach to spin-density matrix from the ballistic to the localized regime. Phys. Rev. B 2005, 71, 195328. [CrossRef]

87. Farokhnezhad, M.; Esmaeilzadeh, M.; Ahmadi, S.; Pournaghavi, N. Controllable spin polarization and spin filtering in a zigzag silicene nanoribbon. J. Appl. Phys. 2015, 117, 173913. [CrossRef]

88. Sun, Q.-F.; Guo, A.-M. Enhanced spin-polarized transport through DNA double helix by gate voltage. Phys. Rev. B 2012, 86, 035424.

89. Martinez, D.F.; Molina, R.A.; Hu, B. Length-dependent oscillations in the dc conductance of laser-driven quantum wires. Phys. Rev. B 2008, 78, 045428. [CrossRef] 